\title{
The Phosphorus/Sulfur Abundance Ratio as a Test of Galactic Cosmic-Ray Source Models
}

\author{
J.S. George*, M.E. Wiedenbeck ${ }^{\dagger}$, W.R. Binns**, E.R. Christian ${ }^{\ddagger}$, A.C. Cummings*, \\ P.L. Hink ${ }^{* *}$, R.A. Leske*, R.A. Mewaldt*, E.C. Stone*, T.T. von Rosenvinge ${ }^{\ddagger}$ and \\ N.E. Yanasak* \\ ${ }^{*}$ California Institute of Technology, Pasadena, CA 91125 USA \\ ${ }^{\dagger}$ Jet Propulsion Laboratory, California Institute of Technology, Pasadena, CA 91109 USA \\ ${ }^{* *}$ Washington University, St. Louis, MO, 63130 USA \\ ${ }^{\ddagger}$ NASA/Goddard Space Flight Center, Greenbelt, MD 20771 USA
}

\begin{abstract}
Galactic cosmic-ray (GCR) elemental abundances display a fractionation compared to solar-system values that appears ordered by atomic properties such as the first ionization potential (FIP) or condensation temperature (volatility). Determining which parameter controls the observed fractionation is crucial to distinguish between GCR origin models. The Cosmic-Ray Isotope Spectrometer (CRIS) instrument on board NASA's Advanced Composition Explorer (ACE) spacecraft can measure the abundances of several elements that break the general correlation between FIP and volatility (e.g., $\mathrm{Na}, \mathrm{P}, \mathrm{K}, \mathrm{Cu}, \mathrm{Zn}, \mathrm{Ga}$, and $\mathrm{Ge}$ ). Phosphorus is a particularly interesting case as it is a refractory (high condensation temperature) element with a FIP value nearly identical to that of its semi-volatile neighbor, sulfur. Using a leaky-box galactic propagation model we find that the $\mathrm{P} / \mathrm{S}$ and $\mathrm{Na} / \mathrm{Mg}$ ratios in the GCR source favor volatility as the controlling parameter.
\end{abstract}

\section{INTRODUCTION}

The origin of the source material for galactic cosmic rays (GCRs) remains one of the unsolved problems of particle astrophysics. Various views support origins as diverse as supernovae ejecta and interstellar dust [1]. The abundances of cosmic-ray elements measured near Earth contain information about the source composition and the effects of transport through the Galaxy. Careful modeling of these propagation effects can be used to determine the source composition and shed light on the sources that contribute to the cosmic rays [2]. The Cosmic-Ray Isotope Spectrometer (CRIS) instrument [3] on board the Advanced Composition Explorer (ACE) spacecraft has been making high-precision measurements of the GCR composition from $\mathrm{He}$ to $\mathrm{Zn}$ at typical energies of $50-500$ $\mathrm{MeV} /$ nucleon.

Elemental GCR abundances display a long-observed elemental fractionation commonly parameterized by the first ionization potential (FIP) [4]. Specifically, elements with FIP values above $\sim 10 \mathrm{eV}$ are depleted in the GCR source by roughly a factor of five relative to solarsystem abundances derived from meteoritic and photospheric observations [5]. A similar dependence on FIP, or possibly on first ionization time (FIT) [see, e.g. 6], has been observed in solar energetic particles, the solar corona, and the solar wind. This similarity led some to suggest that the GCR seed population was originally ejected at $\mathrm{MeV} /$ nucleon energies from the coronae of solar-like stars $[4,7]$. These particles would have been further accelerated at a later time by some other mechanism, widely thought to be passing supernova shock waves [8].

Abundances of mass-59 isotopes measured by ACE essentially rule out the possibility that a supernova can accelerate its own ejecta [9]. The ${ }^{59} \mathrm{Ni}$ atoms in the source decay via electron capture to ${ }^{59} \mathrm{Co}$ with a halflife of $\sim 10^{5}$ years until they are accelerated, when the ions are fully stripped and become essentially stable. The amount of ${ }^{59} \mathrm{Ni}$ in the cosmic rays measures the elapsed time between production and acceleration. ACE data are consistent with the complete decay of all ${ }^{59} \mathrm{Ni}$ in the source. This implies an acceleration time delay greater than $10^{5}$ years, far longer than the $10^{4}$ years required to dissipate the energy from a supernova shock. Compositional arguments based on comparison of the GCR source abundances with those expected from supernova nucleosynthesis have also been used to support the idea of a long delay [10].

Current models envision large associations of OB stars

CP598, Solar and Galactic Composition, edited by R. F. Wimmer-Schweingruber (C) 2001 American Institute of Physics 0-7354-0042-3/01/\$18.00 


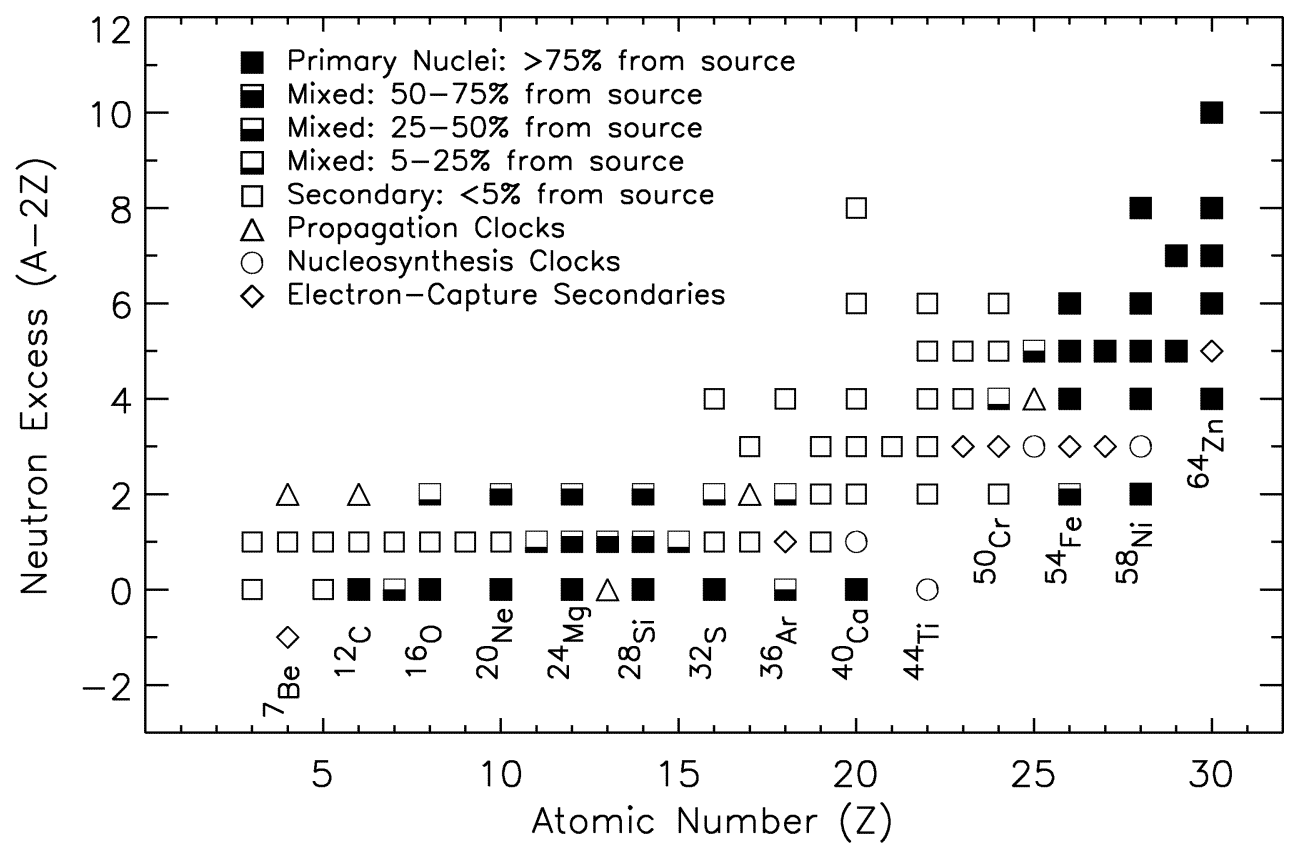

FIGURE 1. Cosmic-ray isotope chart coded according to the fraction of material observed at Earth that came directly from the source. Useful tracer isotopes are the stable nuclides with negligible primary contribution denoted by open squares.

where shock waves from consecutive supernovae sweep out a "superbubble", a region filled with a warm dilute mixture of fresh ejecta and interstellar medium (ISM) material stripped from the walls of the cavity $[11,12]$. Later shocks accelerate material out of the bubble as cosmic rays.

An alternative model is based on the realization that the elemental fractionation may not be controlled by the first ionization potential, but rather by some correlated atomic property such as the condensation temperature, or volatility $[13,14,1]$. In this view, the refractory elements in the cosmic rays are thought to be sputtering products from interstellar dust grains. Weakly charged grains, with their very high mass-to-charge ratios, can easily be accelerated to energies of $\sim 0.1 \mathrm{MeV} /$ nucleon. Energetic ions sputtered off the grains through collisions with the interstellar gas are further accelerated by the same shock, thereby avoiding the need for a second separate acceleration mechanism. The GCR abundances of the refractory elements are set by the physical conditions, such as the temperature, under which the grains condensed. Ions in the gas phase would also be accelerated, accounting for the volatiles in the cosmic rays.

Determining which parameter controls the elemental fractionation is crucial for distinguishing between models of the source. The abundances of several elements that break the general correlation between FIP and volatility can indicate which is actually relevant. Seven of these elements ( $\mathrm{Na}, \mathrm{P}, \mathrm{K}, \mathrm{Cu}, \mathrm{Zn}, \mathrm{Ga}$, and $\mathrm{Ge}$ ) have had abundances measured by the CRIS instrument. The four heavy elements beyond nickel have relatively small corrections due to fragmentation of heavier nuclides. Abundances in this region fall rapidly with increasing nuclear charge so there is little material to contribute to secondary production by fragmentation during transport. These source abundances are reliably determined and limited mainly by statistics [15]. Of these four, $\mathrm{Cu}$ and $\mathrm{Ge}$ favor volatility models. The $\mathrm{Ga}$ abundance is consistent with FIP models with large statistical uncertainty. The volatile element $\mathrm{Zn}$ is depleted by a factor of 2.5 compared to the solar system value, however, as an intermediate-FIP element some depletion is expected in either model.

The source abundances of $\mathrm{Na}$ and $\mathrm{P}$ are limited by uncertainties in the propagation models used to correct for secondary production during transport. Nearly $80 \%$ of the $\mathrm{Na}$ and $\mathrm{P}$ observed at Earth is produced by fragmentation of heavier isotopes. Because of these large corrections, uncertainties in the propagation parameters, particularly in the nuclear fragmentation cross sections, are the dominant sources of error in the derived source abundances.

The Na source abundance has been previously shown to be depleted at the $2 \sigma$ level relative to the solar-system 
abundance [15]. This depletion is consistent with that expected from volatility models. That result is updated here, and the analysis is extended to phosphorus, a somewhat refractory $\left(\mathrm{T}_{c}=1151 \mathrm{~K}\right)$ intermediate-FIP element. The $\mathrm{P} / \mathrm{S}$ ratio is particularly interesting because $\mathrm{P}$ and $\mathrm{S}$ have similar FIP values (10.49 and $10.36 \mathrm{eV}$, respectively) while $\mathrm{S}$ is more volatile $\left(\mathrm{T}_{c}=648 \mathrm{~K}\right)$. A difference in the relative depletions of $\mathrm{S}$ and $\mathrm{P}$ compared to their solar-system values should be a good test of whether FIP or volatility controls the fractionation. The chief difficulty lies in making an accurate determination of the secondary contributions and in assessing the systematic uncertainties in the model.

One approach to the problem of extracting source abundances for elements such as $\mathrm{Na}$ and $\mathrm{P}$ is to use the abundances of purely secondary isotopes, i.e. those produced entirely by fragmentation, as tracers of the amount of secondary production [16]. These tracer isotopes constrain the GCR pathlength that controls the total amount of spallation. Reproducing the measured abundances of isotopes produced by spallation should provide a good estimate of the secondary contribution to ${ }^{23} \mathrm{Na}$ and ${ }^{31} \mathrm{P}$, which have small primary components.

Figure 1 contains an isotope chart coded according to the fraction of material observed at Earth that comes directly from the source. The elements $\mathrm{Na}$ and $\mathrm{P}$ each consist of a single isotope in the cosmic rays. The chart can be used to identify appropriate tracer isotopes, those that are nearby in mass but have even larger secondary contributions than ${ }^{23} \mathrm{Na}$ and ${ }^{31} \mathrm{P}$. We chose seven such isotopes, ${ }^{17} \mathrm{O},{ }^{19} \mathrm{~F},{ }^{21} \mathrm{Ne},{ }^{33} \mathrm{~S},{ }^{36} \mathrm{~S},{ }^{35} \mathrm{Cl}$, and ${ }^{37} \mathrm{Cl}$, for use in this study.

\section{THE ${ }^{23} \mathrm{Na} /{ }^{24} \mathrm{Mg}$ SOURCE RATIO}

The points in Figure 2 show the result of a series of cosmic-ray propagation calculations. The steady-state "leaky-box" model gives equilibrium abundances in a homogeneous Galaxy, accounting for source injection, fragmentation, radioactive decay, energy loss, and particle escape [2]. The five points in each panel represent separate propagation calculations with values for the escape mean free path varying by up to a factor of two. The solid line is a linear fit. In each calculation, the source ratios of isotopes between $\mathrm{Ne}$ and $\mathrm{Fe}$ were adjusted relative to the stable primary isotope ${ }^{24} \mathrm{Mg}$ to reproduce the locally measured values. Data from the CRIS instrument used here were taken over a $\sim 2.5$ year period from $\mathrm{Au}$ gust 1997 through April 2000. The shaded areas plot the percentage difference between the predicted and measured abundance of each tracer isotope compared with the source ratio required to account for the measured value of ${ }^{23} \mathrm{Na} /{ }^{24} \mathrm{Mg}=0.213 \pm 0.003$ in each calculation. $\mathrm{A}$

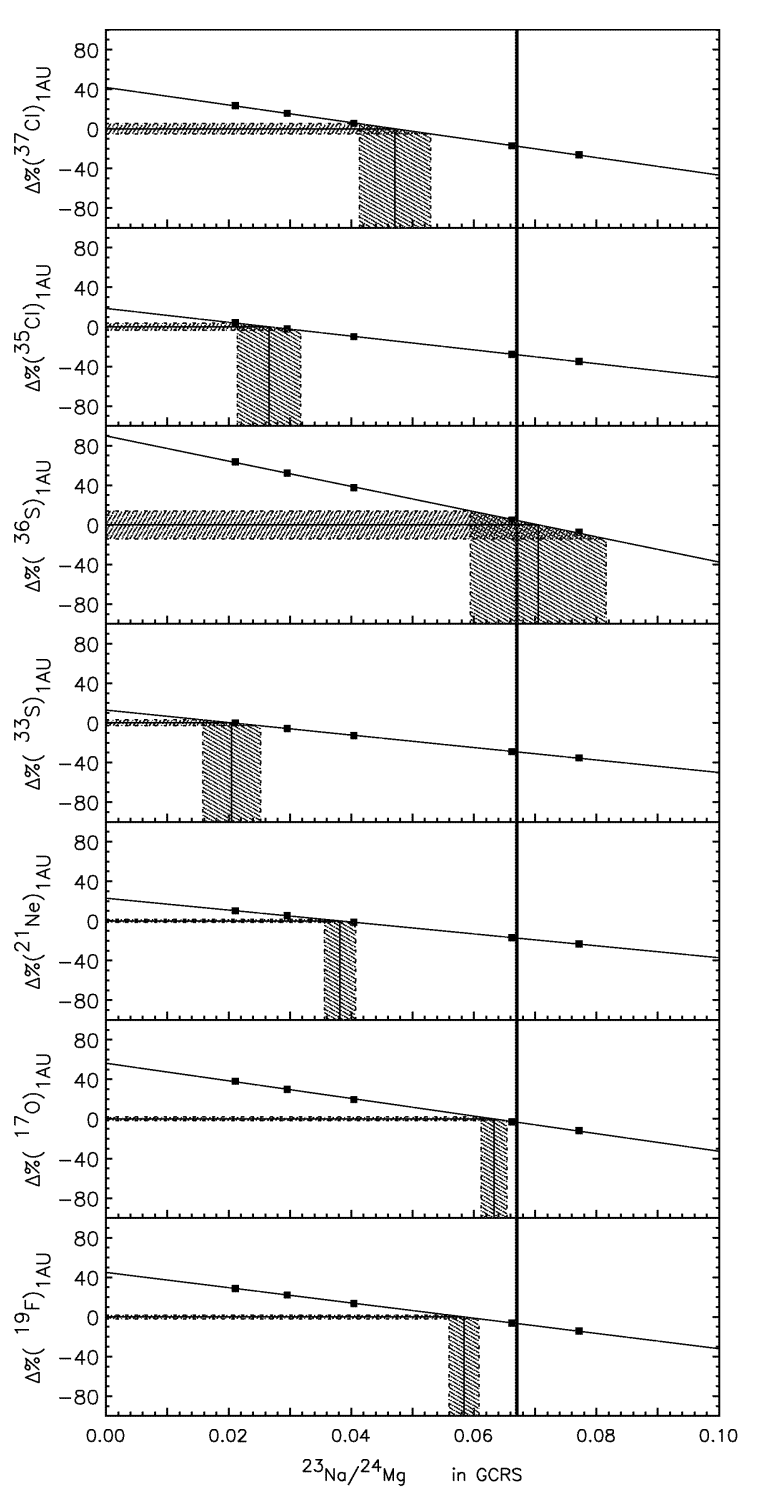

FIGURE 2. Estimates of the ${ }^{23} \mathrm{Na} /{ }^{24} \mathrm{Mg}$ source ratio needed to account for the observed ${ }^{23} \mathrm{Na} /{ }^{24} \mathrm{Mg}$ at Earth in various propagation calculations compared with the abundances of the secondary tracer isotopes predicted by those models. The ordinate in each case is the percent difference between the predicted local abundance and the measured value of the tracer. The vertical line indicates the solar-system ${ }^{23} \mathrm{Na} /{ }^{24} \mathrm{Mg}$ value [5]. Shaded areas indicate statistical uncertainty due to the measurement of the tracer isotope.

model that correctly predicts the measured tracer abundance ( $0 \%$ difference) also provides an estimate of the amount of ${ }^{23} \mathrm{Na}$ in the source. The vertical line shows the solar-system ${ }^{23} \mathrm{Na} /{ }^{24} \mathrm{Mg}$ value of $0.068 \pm 0.005$ [5].

The propagation calculation used to estimate the secondary corrections is a steady-state leaky-box propagation model [17], based on the formalism of Meneguzzi, 
TABLE 1. Relative uncertainties contributing to the estimates for the ${ }^{23} \mathrm{Na} /{ }^{24} \mathrm{Mg}$ source ratio from each of the tracer isotopes.

\begin{tabular}{l|rrrrr}
\hline & \multicolumn{2}{c}{ Statistics } & \multicolumn{2}{c}{ Cross sections } & 年 $\mathrm{Na}$ \\
& Tracer & $\frac{{ }^{23} \mathrm{Na}}{{ }^{24} \mathrm{Mg}}$ & Tracer & ${ }^{23} \mathbf{N a}$ & Source Ratio \\
\hline${ }^{17} \mathrm{O}$ & $4.3 \%$ & $1.4 \%$ & $8.8 \%$ & $11.0 \%$ & $0.063 \pm 0.009$ \\
${ }^{19} \mathrm{~F}$ & $5.1 \%$ & $1.4 \%$ & $9.9 \%$ & $11.0 \%$ & $0.058 \pm 0.009$ \\
${ }^{21} \mathrm{Ne}$ & $8.8 \%$ & $1.4 \%$ & $6.4 \%$ & $11.0 \%$ & $0.038 \pm 0.006$ \\
${ }^{33} \mathrm{~S}$ & $24.6 \%$ & $1.4 \%$ & $6.5 \%$ & $11.0 \%$ & $0.021 \pm 0.006$ \\
${ }^{36} \mathrm{~S}$ & $15.7 \%$ & $1.4 \%$ & $13.7 \%$ & $11.0 \%$ & $0.070 \pm 0.017$ \\
${ }^{35} \mathrm{Cl}$ & $20.4 \%$ & $1.4 \%$ & $10.0 \%$ & $11.0 \%$ & $0.027 \pm 0.007$ \\
${ }^{37} \mathrm{Cl}$ & $12.6 \%$ & $1.4 \%$ & $7.9 \%$ & $11.0 \%$ & $0.048 \pm 0.009$ \\
\hline
\end{tabular}

et al. [2]. Source spectra were power laws in momentum with a spectral index of -2.3 . The interstellar medium $\mathrm{He} / \mathrm{H}$ ratio was assumed to be 0.11 by number with $16 \%$ of the hydrogen in an ionized state [18]. We used the Silberberg and Tsao semi-empirical forms of the nuclear cross sections $[19,20]$ to estimate the amount of fragmentation during transport. The escape mean free path was a function of the velocity $\beta$ and rigidity $R$ with a normalization $\Lambda_{0}$ which is varied in each calculation.

$$
\begin{aligned}
\Lambda_{\mathrm{esc}} & =\Lambda_{0} \beta & & \mathrm{R} \leq 4.9 \mathrm{GV} \\
& =\Lambda_{0} \beta(\mathrm{R} / 4.9 \mathrm{GV})^{-0.7} & & \mathrm{R}>4.9 \mathrm{GV}
\end{aligned}
$$

The effects of solar modulation were taken into account using a spherically symmetric Fisk model [21]. The average modulation level was determined by fitting the $\mathrm{B} / \mathrm{C}$ and $(\mathrm{Sc}+\mathrm{Ti}+\mathrm{V}) / \mathrm{Fe}$ secondary/primary ratios in the CRIS data [see 22]. The appropriate modulation parameter for this time period was determined to be $\phi=460 \mathrm{MV}$.

Table 1 lists the estimates of the ${ }^{23} \mathrm{Na} /{ }^{24} \mathrm{Mg}$ source ratio from each tracer isotope along with the main sources of uncertainty. The first column gives the spread in the estimate due to statistical uncertainty in the measurement of the tracer isotope as shown by the shaded areas in Figure 2. There is also a statistical contribution from the measurement of the ${ }^{23} \mathrm{Na} /{ }^{24} \mathrm{Mg}$ ratio itself, shown in the second column. The third and fourth columns represent uncertainties in the nuclear fragmentation cross sections for the formation of the tracer isotope and ${ }^{23} \mathrm{Na}$ itself. These values are determined by averaging the published uncertainties in the measured cross sections for producing each tracer from all known parents. Each uncertainty is weighted by the relative contribution that that parent makes to the daughter. At least one measurement at an appropriate energy is available for all relevant reactions.

The final column in Table 1 gives the individual estimates for the ${ }^{23} \mathrm{Na} /{ }^{24} \mathrm{Mg}$ source ratio including contributions from each source of uncertainty. Six of the seven estimates are lower than the solar-system values.
The best overall estimate of the ${ }^{23} \mathrm{Na} /{ }^{24} \mathrm{Mg}$ source ratio is taken as a weighted average $\mu$ and its uncertainty $\sigma_{\mu}$ of the individual estimates $\mathrm{x}_{i} \pm \sigma_{i}$ given in Table 1 .

$$
\mu=\frac{\sum\left(\mathrm{x}_{\mathrm{i}} / \sigma_{\mathrm{i}}^{2}\right)}{\sum\left(1 / \sigma_{i}^{2}\right)} \quad \text { and } \quad \sigma_{\mu}^{2}=\frac{1}{\sum\left(1 / \sigma_{\mathrm{i}}^{2}\right)}
$$

Some caution is warranted as this approach treats the individual uncertainties as being completely uncorrelated. In reality some contributions are highly correlated, such as that from the cross section for producing ${ }^{23} \mathrm{Na}$. The combined uncertainty addresses the spread in the individual measurements but is not sensitive to a systematic shift.

The quality of the fit is estimated with a test statistic $\chi^{2}=\Sigma\left(\mathrm{x}_{\mathrm{i}}-\mu\right)^{2} / \sigma_{\mathrm{i}}^{2}$. Determining the mean from seven measurements leaves six remaining degrees of freedom, giving a reduced $\chi^{2}$ value $\chi_{v}^{2}=4.7$. Such a large value points out that there are non-statistical fluctuations which are not represented by the stated uncertainties in Figure 2 and Table 1. These may suggest that the actual uncertainties in the cross-section measurements are larger than those ascribed to them in the literature. One way to characterize this additional uncertainty is to increase the uncertainty of the mean by an amount needed to make $\chi_{v}^{2}=1$, that is, $\sigma=\chi_{\nu} \sigma_{\mu}$.

With this approach we obtain an overall best value for $\left({ }^{23} \mathrm{Na} /{ }^{24} \mathrm{Mg}\right)_{G C R S}$ of $0.038 \pm 0.006$. Data from the Ulysses spacecraft [23] supports the use of solar-system isotopic abundances [5] to determine the elemental $\mathrm{Na} / \mathrm{Mg}$ ratio in the source. We find the $\mathrm{Na} / \mathrm{Mg}$ ratio in the GCR source relative to the solar system to be:

$$
(\mathrm{Na} / \mathrm{Mg})_{\mathrm{GCRS}} /(\mathrm{Na} / \mathrm{Mg})_{\mathrm{SS}}=0.57 \pm 0.11
$$

This result is consistent with our previous result of $0.62 \pm 0.19$ [15] using only the three lightest tracers taking the full spread of the individual estimates as a measure of the uncertainty. That calculation used the Webber et al. cross section parameterization [24] and a somewhat higher solar modulation level.

\section{THE ${ }^{31} \mathbf{P} /{ }^{32}$ S SOURCE RATIO}

The same analysis has also been carried out for the source ${ }^{31} \mathrm{P} /{ }^{32} \mathrm{~S}$ ratio. In this case, sulfur is known to be depleted relative to the solar-system value. The question being investigated is whether $\mathrm{P}$ and $\mathrm{S}$ are depleted by the same amount as would be expected from a FIPcontrolled fractionation.

The results of the calculations are displayed in Figure 3. As with ${ }^{23} \mathrm{Na} /{ }^{24} \mathrm{Mg}$, only the statistical uncertainty due to measurement of the tracer isotope is shown. The measured ${ }^{31} \mathrm{P} /{ }^{32} \mathrm{~S}$ value near Earth is $0.268 \pm 0.010$. A 
TABLE 2. Relative uncertainties contributing to the ${ }^{31} \mathrm{P} /{ }^{32} \mathrm{~S}$ source ratio estimates from each of the tracer isotopes.

\begin{tabular}{|c|c|c|c|c|c|}
\hline & \multicolumn{2}{|c|}{ Statistics } & \multicolumn{2}{|c|}{ Cross sections } & \multirow{2}{*}{$\begin{array}{c}{ }^{\frac{31}{32} \mathrm{P}} \\
\text { Source Ratio }\end{array}$} \\
\hline & Tracer & $\frac{{ }^{31} \mathrm{p}}{{ }^{32} \mathrm{~S}}$ & Tracer & ${ }^{31} \mathbf{P}$ & \\
\hline${ }^{17} \mathrm{O}$ & \multicolumn{2}{|c|}{$4.1 \% 3.6 \%$} & $8.8 \%$ & $5.0 \%$ & $0.102 \pm 0.012$ \\
\hline${ }^{19} \mathrm{~F}$ & \multicolumn{2}{|c|}{$5.1 \% 3.6 \%$} & $9.9 \%$ & $5.0 \%$ & $0.095 \pm 0.012$ \\
\hline${ }^{21} \mathrm{Ne}$ & \multicolumn{2}{|c|}{$8.8 \% 3.6 \%$} & $6.4 \%$ & $5.0 \%$ & $0.062 \pm 0.008$ \\
\hline${ }^{33} \mathrm{~S}$ & \multicolumn{2}{|c|}{$24.3 \% 3.6 \%$} & $6.5 \%$ & $5.0 \%$ & $0.034 \pm 0.009$ \\
\hline${ }^{36} \mathrm{~S}$ & \multicolumn{2}{|c|}{$15.6 \% 3.6 \%$} & $13.7 \%$ & $5.0 \%$ & $0.114 \pm 0.025$ \\
\hline${ }^{35} \mathrm{Cl}$ & \multicolumn{2}{|c|}{$20.2 \% 3.6 \%$} & $10.0 \%$ & $5.0 \%$ & $0.044 \pm 0.010$ \\
\hline${ }^{37} \mathrm{Cl}$ & \multicolumn{2}{|c|}{$12.6 \% 3.6 \%$} & $7.9 \%$ & $5.0 \%$ & $0.077 \pm 0.012$ \\
\hline
\end{tabular}

vertical line represents the solar-system ${ }^{31} \mathrm{P} /{ }^{32} \mathrm{~S}$ ratio [5] of $0.021 \pm 0.003$, expected if the depletion is the same. It is clear that all of the seven tracer isotopes predict a ${ }^{31} \mathrm{P} /{ }^{\beta 2} \mathrm{~S}$ ratio higher than that of the solar system, i.e., that ${ }^{31} \mathrm{P}$ is less depleted than ${ }^{32} \mathrm{~S}$ in the GCR source.

The individual estimates are given in Table 2 along with the corresponding contributions to their uncertainties. Using the same approach as before, we combine them into an overall best value of $\left({ }^{31} \mathrm{P} /{ }^{32} \mathrm{~S}\right)_{G C R S}$ $=0.065 \pm 0.01$. The reduced $\chi^{2}$ value folded into the uncertainty estimate was 6.3 . This best estimate represents an enhancement of $3.1 \pm 0.7$ over the solar-system value. Pending completion of the CRIS results for the $\mathrm{S} / \mathrm{Mg}$ source ratio, we use the HEAO-3-C2 [25] value of $0.126 \pm 0.009$ to find

$$
(\mathrm{P} / \mathrm{Mg})_{\mathrm{GCRS}} /(\mathrm{P} / \mathrm{Mg})_{\mathrm{SS}}=0.80 \pm 0.19
$$

\section{DISCUSSION AND CONCLUSION}

Using a secondary tracer isotope technique, we estimated the source abundances of ${ }^{23} \mathrm{Na}$ and ${ }^{31} \mathrm{P}$ based on new data from CRIS. An estimate based on seven tracer isotopes suggests that the semi-volatile element $\mathrm{Na}$ is depleted in the GCR source relative to the more refractory $\mathrm{Mg}$, compared to solar-system abundances. This result is consistent with a prior analysis of $\mathrm{Na}$ based on three tracer isotopes [15]. Similarly, the less volatile $P$ is enhanced with respect to the highly volatile $\mathrm{S}$ by a factor of three, even though each has a nearly identical FIP value. The $\mathrm{P} / \mathrm{Mg}$ source ratio is consistent with the solar-system value or a small depletion due to the difference in condensation temperatures. It should be noted, however, that correlated errors such as incorrect cross sections for producing $\mathrm{Na}$ or $\mathrm{P}$ could systematically shift the individual estimates. This is a concern with the large corrections for secondary production that are used here.

Figure 4 summarizes the current results. The source abundance of each element relative to its solar-system

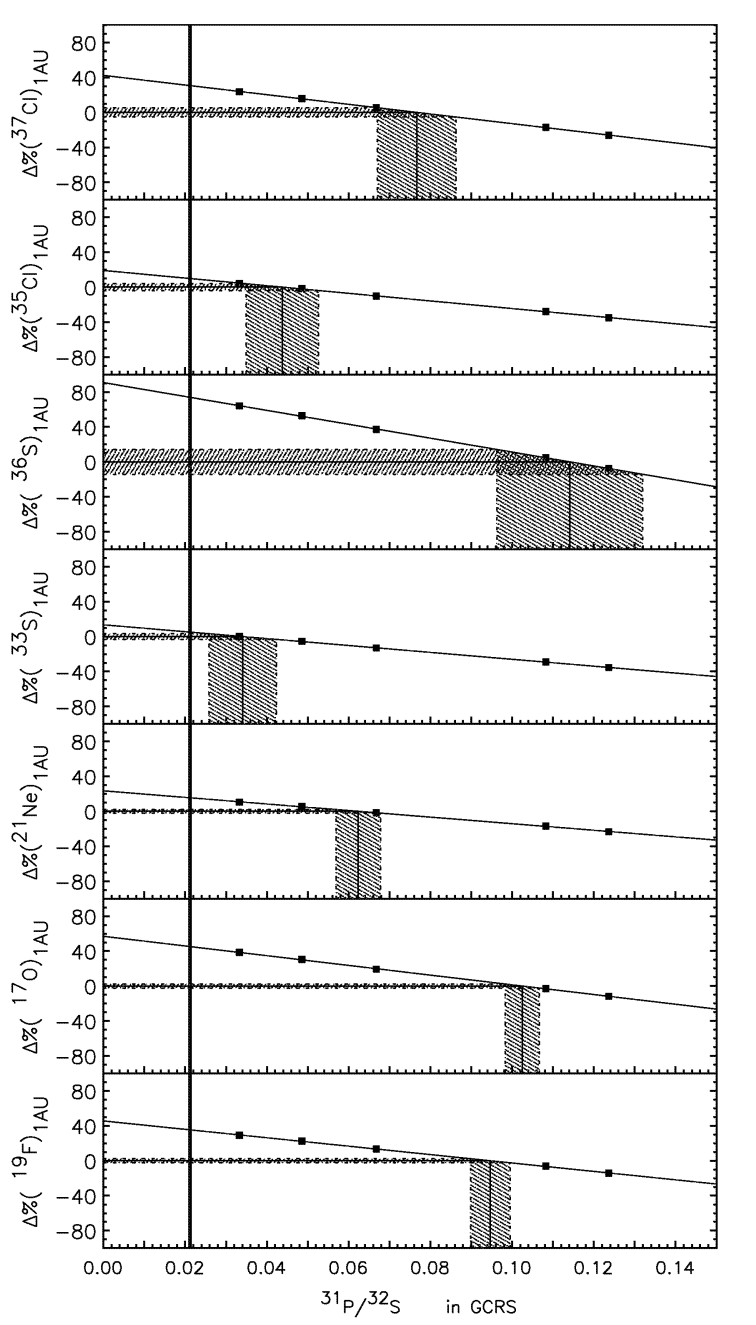

FIGURE 3. Estimates of the ${ }^{31} \mathrm{P} /{ }^{32} \mathrm{~S}$ source ratio from various tracer isotopes as described in Figure 2 The vertical line indicates the solar-system ${ }^{31} \mathrm{P} /{ }^{32} \mathrm{~S}$ value [5]. Shaded areas show statistical uncertainty from measurement of the tracer isotope.

value is plotted as a function of first ionization potential, normalized to $\mathrm{Mg} \equiv 1$. One possible FIP parameterization has been overlaid on the plot. The filled squares indicate the present results for $\mathrm{Na}$ and $\mathrm{P}$ while the rightpointing triangles represent previous results from CRIS data [15], and the crosses indicate data from the HEAO3-C2 spacecraft [25] for context. The low values of $\mathrm{Na} / \mathrm{Mg}, \mathrm{Cu} / \mathrm{Fe}$, and $\mathrm{Ge} / \mathrm{Fe}$, and the enhancement of $\mathrm{P} / \mathrm{S}$ are consistent with expectations of a volatility model. The overabundance of $\mathrm{C}$ may reflect additional sources of material unrelated to the present investigation [1].

Since $\mathrm{Zn}$ and $\mathrm{S}$ have nearly the same condensation temperature the apparent low $\mathrm{S} / \mathrm{Zn}$ ratio seems at first to support FIP. Volatility models explain the low sulfur as a consequence of a rigidity dependence of the shock acceleration efficiency [1]. Volatile elements such 


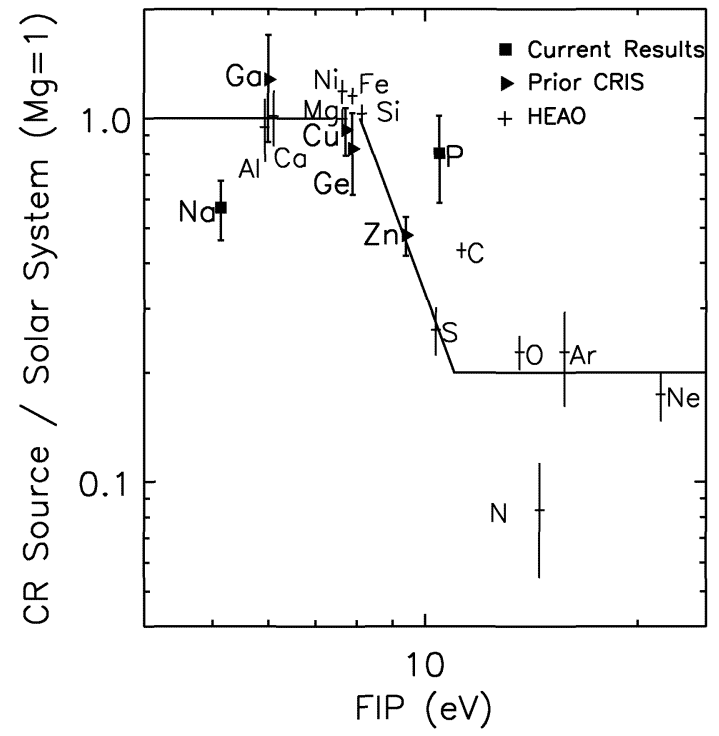

FIGURE 4. GCR source abundances relative to solar system values are plotted as a function of the first ionization potential. Filled squares are from the current work. Right-pointing triangles are prior CRIS results [15], crosses indicate results from HEAO-3-C2 [25]. The solid line represents one possible parameterization of the FIP step.

as $\mathrm{S}$ and $\mathrm{Zn}$ are accelerated largely out of the gas phase. A cool photo-ionized gas with a mass-to-charge ratio $A / Q \sim A^{0.7}$ could account for a $\mathrm{S} / \mathrm{Zn}$ ratio of $\sim 0.6 \mathrm{com}$ pared to the solar system value. A similar, weaker, mass bias in the acceleration efficiency for even refractory elements is used to explain the excess of $\mathrm{Fe}$ and $\mathrm{Ni}$ in the source relative to $\mathrm{Al}, \mathrm{Ca}, \mathrm{Mg}$, and $\mathrm{Si}$. The Se source abundance would be an interesting test of a mass dependence. Se is heavier than $\mathrm{Zn}$, but has a very similar FIP and condensation temperature. CRIS may be able to address this issue once the cosmic-ray fluxes return to their high solar minimum levels allowing for greater statistics.

A pattern seems to be emerging that supports volatility as the relevant parameter for the elemental fractionation of galactic cosmic rays. However, caution is warranted in assessing the significance of these results. Nonstatistical fluctuations in the individual estimates are at least as large as the statistical uncertainties. The approach taken here is one quantitative method of characterizing the measurements but may still underestimate the actual spread. At the same time, these results do provide a stronger indication that some deficit of $\mathrm{Na} / \mathrm{Mg}$ and some enhancement of $\mathrm{P} / \mathrm{S}$ compared to the solar-system values is present in the galactic cosmic-ray source. Completion of the CRIS results for the primary elements will help place these results in context, but significant improvements for $\mathrm{Na}$ and $\mathrm{P}$ may require more precise measurements of the fragmentation cross sections.

\section{ACKNOWLEDGMENTS}

This work was supported by NASA at the California Institute of Technology (under grant NAG5-6912), the Jet Propulsion Laboratory, the Goddard Space Flight Center, and Washington University.

\section{REFERENCES}

1. Meyer, J., Drury, L., and Ellison, D., ApJ, 487, 182-196 (1997).

2. Meneguzzi, M., Audouze, J., and Reeves, H., $A \& A, \mathbf{1 5}$, 337 (1971).

3. Stone, E., et al., Space Sci. Rev., 96, 285-356 (1998).

4. Cassé, M., and Goret, P., ApJ, 221, 703 (1978).

5. Anders, E., and Grevesse, N., Geochim. Cosmochim. Acta, 53, 197-214 (1989).

6. Geiss, J., Space Sci. Rev., 85, 241-252 (1998).

7. Meyer, J.-P., ApJS, 57, 173-204 (1985).

8. Ginzburg, V., and Syrovatskii, S., The Origin of Cosmic Rays, Pergamon Press, Oxford, England, 1964.

9. Wiedenbeck, M., et al., ApJL, 523, L61-L64 (1999).

10. Meyer, J., and Ellison, D., "The Origin of the Present Day Cosmic Rays (I)", in LiBeB, Cosmic Rays, and Related Xand Gamma-Rays, edited by R. Ramaty et al., ASP Conf. Series \#171 (Astr. Soc. of the Pacific), San Francisco, 1999, pp. 187-206.

11. Higdon, J., Lingenfelter, R., and Ramaty, R., ApJ, 509, L33-L36 (1998).

12. Parizot, E., and Drury, L., A\&A, 349, 673-684 (1999).

13. Bibring, J.-P., and Cesarsky, C., Proc. $17^{\text {th }}$ ICRC (Paris), 2, 289 (1981).

14. Epstein, R., MNRAS, 193, 723 (1980).

15. George, J., Wiedenbeck, M., Barghouty, A., et al., "Cosmic Ray Source Abundances and the Acceleration of Cosmic Rays", in [26], pp. 437-440.

16. Stone, E., and Wiedenbeck, M., ApJ, 231, 606-623 (1979).

17. Leske, R., ApJ, 405, 567-583 (1993).

18. Soutoul, A., Ferrando, P., and Webber, W., Proc. $21^{\text {st }}$ ICRC, 3, 337 (1990).

19. Silberberg, R., Tsao, C., and Barghouty, A., ApJ, 501, 911 (1998).

20. Tsao, C., Silberberg, R., and Barghouty, A., ApJ, 501, 920 (1997).

21. Fisk, L., J. Geophys. Res., 76, 221 (1971).

22. Davis, A., Mewaldt, R., et al., "On the Low Energy Decrease in Galactic Cosmic Ray Secondary/Primary Ratios", in [26], pp. 421-424.

23. Connell, J., and Simpson, J., Proc. $23^{\text {rd }}$ ICRC (Calgary), 1, 559 (1993).

24. Webber, W., Kish, J., and Schrier, D., Phys Rev C, 41, 566-571 (1990).

25. Engelmann, J., et al., Astron. Astrophys., 233, 96-111 (1990).

26. Mewaldt, R., et al., editors, Acceleration and Transport of Energetic Particles Observed in the Heliosphere, AIP Conference Series \#528, New York, 2000. 